Original Paper http://ajol.info/index.php/ijbcs http://indexmedicus.afro.who.int

\title{
Confirmation de QTL et validation de marqueurs SNPs associés à la résistance du niébé à Colletotrichum capsici, agent responsable de la maladie des taches brunes
}

\author{
Ibié G. THIO ${ }^{1,2 *}$, Nofou OUEDRAOGO ${ }^{1}$, Serge W.F.M. ZIDA ${ }^{1}$, \\ Joseph T.B. BATIENO ${ }^{1}$, Elisabeth P. ZIDA ${ }^{2}$, Jean Baptiste TIGNEGRE ${ }^{1}$, \\ Jeremy T. OUEDRAOGO ${ }^{1}$, Mahamadou SAWADOGO ${ }^{1,3}$, Paco SEREME ${ }^{2}$, \\ Erik W. OHLSON ${ }^{4}$ et Michael P. TIMKO
}
${ }^{1}$ Laboratoire de Génétique et Biotechnologie, Institut de l'Environnement et de Recherches Agricoles (INERA), Ouagadougou 01, BP 476, Ouagadougou, Burkina Faso.
${ }^{2}$ Laboratoire de Phytopathologie, Institut de l'Environnement et de Recherches Agricoles (INERA), Ouagadougou 01, BP 476, Ouagadougou, Burkina Faso.
${ }^{3}$ Laboratoire de Biosciences, Université Joseph Ki-Zerbo, Ouagadougou, Burkina Faso.
${ }^{4}$ Department of Biology, University of Virginia, Charlottesville, VA, 22904, USA.
*Auteur correspondant ; E-mail : gilthiolpr@gmail.com

\begin{tabular}{ccc}
\hline Received: 30-04-2021 & Accepted: 07-06-2021 & Published: 30-06-2021 \\
\hline
\end{tabular}

\section{RESUME}

Le niébé (Vigna unguiculata (L.) Walp.) est une légumineuse à graine très importante et constitue la principale source de protéines végétales pour l'alimentation des populations d'Afrique Subsaharienne. Sa production au Burkina Faso est entravée par la maladie des taches brunes provoquée par un champignon, Colletotrichum capsici (Syd.) Butler et Bisby. C'est dans la perspective d'accrô̂tre la productivité du niébé que nous avons entrepris de renforcer la lutte variétale contre cet agent pathogène. L'identification de marqueurs SNPs (Single Nucleotide Polymorphism) et QTL liés à la résistance à la maladie des taches brunes a été entrepris à partir d'une population biparentale $\mathrm{F}_{2}$ issus du croisement entre la variété sensible Tiligré et celle résistante KN-1. L'analyse QTL de la résistance du niébé à $C$. capsici à partir de la méthode ICIM add. a permis de confirmer et de valider respectivement un QTL majeur dénommé qBBDR2.1 et 9 marqueurs SNPs convertis, lesquels ont été cartographiés sur le chromosome Vu02 du niébé. Ce QTL dominant a présenté des effets additifs élevés liés aux allèles favorables de KN-1 et des valeurs de PVE de l'ordre de 51,50\% et 55,33\%, respectivement aux $21^{\text {ème }}$ et $28^{\text {ème }}$ JAI.

(C) 2021 International Formulae Group. All rights reserved.

Mots clés: Niébé, taches brunes, résistance, marqueurs SNPs, QTL, Burkina Faso.

\section{Confirmation of QTL mapping and validation of SNPs markers associated to cowpea resistance to Colletotrichum capsici, causal agent of brown blotch disease}

\section{ABSTRACT}

Cowpea (Vigna unguiculata (L.) Walp.) is one of the most important grain legume crops and constitutes the main source of plant protein for people food in sub-Saharan Africa. Cowpea production in Burkina Faso is 
constrained by brown blotch disease caused by a fungal, Colletotrichum capsici (Syd.) Butler and Bisby. In order to increase cowpea productivity we initiated a project to enhance host plant resistance to control the pathogen. The identification of SNP (Single Nucleotide Polymorphism) markers and QTL associated with brown blotch disease resistance was undertaken from a bi-parental $F_{2}$ population resulting from a cross between the sensitive variety Tiligre and the resistant $\mathrm{KN}-1$ to the disease. QTL analysis of cowpea resistance to $C$. capsici using the ICIM add method. Allowed to confirm and validate respectively a major QTL named $q B B D R 2.1$ and 9 converted SNP markers, which were mapped on cowpea chromosome Vu02. This dominant QTL showed higher additive effects associated to alleles from $\mathrm{KN}-1$ and PVE values of $51.50 \%$ and $55.33 \%$ respectively at 21 and 28 days after inoculation.

(C) 2021 International Formulae Group. All rights reserved.

Keywords: Cowpea, brown blotch, resistance, SNP markers, QTL, Burkina Faso.

\section{INTRODUCTION}

Le niébé (Vigna unguiculata (L.) Walp.) est l'une des plus importantes légumineuses à graines pour l'alimentation des populations des pays de l'Afrique subsaharienne (Singh et al., 2002; Smýkal et al., 2015). Il est généralement cultivé dans les zones arides des régions tropicales et subtropicales (Halime et al., 2014). Le niébé constitue une source considérable de protéines végétales pour l'alimentation de nombreuses populations (Singh et al., 2002). La production mondiale du niébé en 2016 s'élevait à 6991174 de tonnes de grains secs produites sur une superficie de plus de 12316878 d'hectares (FAOSTAT, 2016). L'Afrique subsaharienne est la principale zone de production du niébé avec $94 \%$ de la production mondiale (Nedumaran et al., 2015).

Le Burkina Faso, pays sahélien, est le troisième pays producteur mondial de niébé après le Nigeria (3027596 tonnes/an) et le Niger (1987100 tonnes/ an). La production globale en grains du niébé au Burkina Faso en 2016 représente 603635 tonnes (FAOSTAT, 2017). La contribution annuelle du niébé au PIB burkinabè est estimée entre 19 et 22 milliards de Francs CFA en moyenne (INERA, 2003). En 2010, le revenu monétaire tiré du niébé représentait près de $2,1 \%$ du PIB global du pays (Dabat et al., 2012). Le niébé constitue ainsi une source très importante de revenus monétaires pour les producteurs et les transformateurs (Ouédraogo, 2003). Il occupe de ce fait une place très importante dans la résilience des ménages pauvres et constitue un véritable secours alimentaire, notamment pendant les périodes de soudure.
En dépit de son rôle très important dans la sécurité alimentaire, des baisses de rendements sont constatées chaque année dans les différents systèmes de production du niébé. Au Burkina Faso et dans bien d'autres pays en Afrique, la production du niébé est confrontée à des contraintes multiples et d'origines diverses. Parmi ces contraintes, figure la maladie des taches brunes causée par Colletotrichum capsici qui constitue l'un des facteurs limitant la production du niébé (Begum et al., 2008). Cette maladie fongique occasionne chaque année des pertes importantes de rendement pouvant atteindre $100 \%$ chez les variétés sensibles de niébé (Sérémé et al., 2001; Adegbite et Amusa, 2008). Malgré la multitude de méthodes de lutte utilisées, la maladie des taches brunes continue de causer des dégâts énormes en compromettant sérieusement les politiques de développement en termes de sécurité alimentaire. Cependant, l'utilisation de variétés résistantes de niébé à la maladie des taches brunes combinée avec des pratiques agricoles appropriées, est une stratégie très efficace et durable pour contrôler la maladie (Enyiukwu et al., 2014; Obi et Barriuso-vargas, 2014).

Les progrès technologiques dans le domaine de la biologie moléculaire ont permis le développement de marqueurs moléculaires à haut débit. Ces marqueurs sont généralement associés aux gènes contrôlant la résistance à des maladies, à la sécheresse, aux plantes parasites ou autres ravageurs comme les insectes nuisibles. Ils sont le plus souvent utilisés pour des études de diversité génétique, de caractères, la cartographie des gènes d'intérêts, la recherche de QTL en vue de la 
sélection assistée par marqueurs, etc. Chez le niébé, plusieurs marqueurs moléculaires dont les RFLP, les AFLP, les SCAR, les SSR et, récemment, les SNPs ont été développés. Les investigations de Muchero et al. (2011) ont permis d'identifier à partir de marqueurs SNPs polymorphes un QTL majeur lié à la résistance à Macrophomina Phaseolina. De même, les travaux de Pottorff (2012) ont permis la construction d'une carte de liaison génétique de la résistance du niébé aux races 3 et 4 de Fusarium oxysporium et de Macrophomina Phaseolina, à partir de marqueurs SNPs polymorphes. Récemment, Adetumbi et al. (2016), en travaillant sur la maladie des taches brunes au Nigeria, ont identifié et cartographié des marqueurs SNPs polymorphes chez les variétés IT-95K-193-12 et Ife-Brown. Collard et al. (2006) ont, quant à eux, confirmé et validé respectivement des QTLs et marqueurs SSRs liés à la résistance à Fusarium pseudograminearum, responsable de la maladie de la pourriture de la couronne du blé. Ohlson et al. (2018) ont aussi cartographié 4 QTLs dont le qBBDR1 est associé à la résistance à $C$. capsici chez le niébé.

La présente étude a pour objectif de confirmer un QTL lié à la résistance du niébé à C. capsici en vue de la sélection assistée par marqueurs. Ce QTL a été détecté sur le chromosome 2 du niébé à partir de l'analyse d'association entre des marqueurs SNPs polymorphes et le caractère de résistance au sein d'une population biparentale (Thio, 2018). Les objectifs spécifiques ont été, entre autres, de préciser la position du QTL de résistance, d'estimer ses effets, de tester sa stabilité et d'identifier les régions génomiques impliquées dans la résistance à $C$. capsici.

\section{MATERIEL ET METHODES}

\section{Phénotypage de la population $F_{2}$ de confirmation}

La population $F_{2}$ utilisée pour la confirmation du QTL de résistance a été évaluée en condition contrôlée à l'Université de Virginie aux États-Unis d'Amérique de décembre 2016 à janvier 2017. Deux cents individus $\mathrm{F}_{2}$ et leurs deux parents Tiligré (10 individus) et $\mathrm{KN}-1$ (10 individus), ainsi que des
$\mathrm{F}_{1}$ (4 individus), ont été utilisés pour cette étude. Les semences issues des individus $\mathrm{F}_{2}, \mathrm{~F}_{1}$ et de leurs parents ont été traitées artificiellement à l'hypochlorite de sodium $10 \%$ pendant $5 \mathrm{mn}$ selon les recommandations de Timko Lab. Les semis ont été réalisés dans des pots (dimension $17 \times 11 \mathrm{~cm}$ ) contenant de la terre stérilisée à raison d'une graine par pot. Les plantules âgées de 21 jours ont été inoculées par l'isolat C. cap-SA (variant 1 de C. capsici) par la technique d'aspersion sur plantules. Les observations ont été faites aux $7^{\text {ème }}, 14^{\text {ème }}, 21^{\text {ème }}$ et $28^{\text {ème }} \mathrm{JAI}$ en utilisant une échelle de notation de cinq classes (1 à 5) avec les notes $1=$ aucun symptôme, $2=$ présence de petites taches brunes sur la tige, $3=$ taches coalescentes sur la tige, $4=$ taches coalescentes avec présence d'acervules sur la tige, mais plantes non mortes, et $5=$ tiges flétries, plantes mortes. Pour la désignation des individus en classe de sensibilité, l'incidence et la sévérité de la maladie ont été utilisées comme critère de classification. Pour l'incidence de la maladie, les individus ayant la note 1 ont été considérés comme résistants et ceux ayant les notes 2, 3, 4 et 5 comme sensibles. Pour l'indice de sévérité, les notes 1 et 2 ont été affectées aux individus résistants tandis que les individus ayant les notes 3, 4 et 5 sont ceux sensibles. L'aire en dessous de la courbe de progression de la maladie (ADCPM) ou Area Under the Disease Progress Curve (AUDPC) de la maladie a été déterminée à partir des notes de sévérité de la maladie selon le modèle de Shaner et Finney (1977).

$\operatorname{AUDPC}(\mathbf{A D C P M})=\sum_{\mathrm{i}=1}^{\mathrm{n}}\left[\left(\mathbf{Y}_{\mathbf{i}+\mathbf{1}}+\mathbf{Y}_{\mathbf{i}}\right) / \mathbf{2}\right] *\left(\mathbf{t}_{\mathbf{i}+\mathbf{1}}-\mathbf{t}_{\mathbf{i}}\right)$

Où $n=$ nombre total d'observations, $Y_{i}$ et $Y_{i+1}$ représentent des mesures de l'indice moyen de la sévérité de la maladie respectivement aux temps ti et $t_{i+1}$ avec $\left(t_{i+1}-t_{i}\right)$ représentant l'intervalle de temps qui sépare deux observations successives.

Les scores (ou notes) de la maladie, l'incidence, l'indice de sévérité (ou sévérité) et l'AUDPC ont été utilisés comme paramètres de résistance pour la détection de QTL. 


\section{Extraction des ADN génomiques}

Deux à trois jeunes feuilles âgées de 3 à 5 jours de chaque individu ont été prélevées et séchées sous étuve pendant 48 heures à $37^{\circ} \mathrm{C}$. L'ADN génomique de chaque individu a été obtenu à partir de $25 \mathrm{mg}$ de matière sèche par la méthode CTAB (Doyle and Doyle, 1987). Les concentrations de l'ADN ont été ajustées à $25 \mu \mathrm{g} / \mathrm{ml}$ à l'aide de tampon TE, et l'ADN a été placé dans des plaques de 96 puits, puis stockés à $4{ }^{\circ} \mathrm{C}$.

\section{Réaction PCR et électrophorèse des produits}

La réaction $\mathrm{PCR}$ a été réalisée selon le modèle Allele-Specific PCR (AS-PCR) (Tableau 1) décrit par Gaudet et al. (2009) dont la particularité est l'emploi d'un triplé d'amorces composé de deux amorces spécifiques correspondant à chacun des allèles polymorphes des deux parents. La troisième amorce, commune, est l'amorce flanquant la région génomique à amplifier.

La réaction PCR a été réalisée dans un volume final de $20 \mu \mathrm{l}$ constitué d'un mélange de 1,5 $\mu$ l d'ADN, 1x tampon PCR (10nM Tris HCL, $50 \mathrm{mM} \mathrm{KCl}, 1,5 \mathrm{mM} \mathrm{MgCl} 2), 0,2 \mathrm{mM}$ de dNTP, $1 \mu 1$ de chaque amorce à $10 \mathrm{mM}, 1$ $\mu 1$ de Taq Polymérase, du $\mathrm{MgCl} 2$ et d'eau distillée. Les cycles de températures pour la réaction PCR ont été réalisés dans un thermocycleur de type T100 Thermal Cycler.

Le programme PCR utilisé comprend une étape de dénaturation initiale à $94^{\circ} \mathrm{C}$, pendant $3 \mathrm{mn}$, suivi de 35 cycles successifs de dénaturation à $94{ }^{\circ} \mathrm{C}$, pendant $30 \mathrm{~s}$, d'hybridation à $55^{\circ} \mathrm{C}$ ou $58^{\circ} \mathrm{C}$, respectivement pour les marqueurs A2_00591, A2_01793, A2_14997, A2_04189, A2_32056 et A2_21071, et A2_02471, A2_13136 et A2_03535, pendant 30 s, et d'élongation à 72 ${ }^{\circ} \mathrm{C}$, pendant $1 \mathrm{mn} 30 \mathrm{~s}$. L'élongation finale a été effectuée à $72{ }^{\circ} \mathrm{C}$, pendant $5 \mathrm{mn}$.

Huit à $10 \mu \mathrm{l}$ de chaque produit PCR ont été utilisés pour la migration électrophorétique. Ainsi, les marqueurs A2_02471, A2_03535, A2_32056, A2_01793 et A2_13136 ont été isolés sur un gel d'agarose $8 \%$ (contenant 2,5 $\mu 1 / 1$ de bromure d'éthidium) dans du TAE à 240 $\mathrm{V}$, pendant $2 \mathrm{~h} 30 \mathrm{mn}$. Les marqueurs
A2_00591, A2_14997, A2_04189 et A2_2107 ont été détectés sur un gel de polyacrylamide 6\% (PAGE). Les bandes obtenues ont été visualisées sous une transillumination $\mathrm{UV}$.

\section{Analyse QTL}

Neuf marqueurs SNPs candidats ont été testés sur 200 individus $\mathrm{F}_{2}$ et l'analyse d'association marqueur-phénotype a été réalisée grâce au modèle ICIM add. du logiciel QTLIciMapping version 4.1. L'ensemble des 200 génotypes $F_{2}$ a fait l'objet d'un filtre en vue d'exclure de l'analyse les individus chez lesquels la fréquence de recombinaison génétique entre deux marqueurs adjacents dépasse la normale. Cela permet d'améliorer la qualité du QTL détecté et d'éliminer les cas de distorsions de ségrégation. Collard et al. (2005) ont défini des valeurs du pourcentage de variance phénotypique expliqué (PVE) supérieures à $10 \%$ pour déclarer un QTL majeur. Compte tenu des conditions environnementales instables qui peuvent amplifier les effets de QTLs détectés, le seuil proposé par Bratteler (2006), des valeurs du PVE supérieures à $25 \%$, a été retenu dans la présente étude pour déclarer un QTL majeur.

\section{Cartographie de QTL et marqueurs SNPs associés}

Dans le but de situer le QTL de résistance et les marqueurs SNPs sur le chromosome 2, une cartographie de population biparentale a été réalisée, en employant le rapport de maximum de vraisemblable (LOD) et en utilisant la fonction de cartographie de Konsambi. La valeur du LOD (LOD score seuil) pour laquelle la présence d'un QTL est détectée a été déterminée à 1000 permutations (Churchill et Doerge, 1994) pour les quatre paramètres de la résistance. En cas de présence d'un QTL (test de significance, $\mathrm{P}<0,05$ ), la valeur du LOD score max est supérieure à celle du LOD score seuil. Le programme MAP (Linkage map construction in biparental population) du logiciel QTLIciMapping a été utilisé pour la construction de la carte génétique. Le programme Mapchart a également été utilisé pour générer des cartes 
génétiques à partir des données issues du programme MAP (Voorrips, 2002).

Région génomique et analyse fonctionnelle des gènes de résistance

Les potentiels gènes de résistance impliqués dans la résistance à la maladie des taches brunes du niébé ont été identifiés à partir de l'annotation du programme Phytozome (WGS, Vunguiculata_469_v1.1.annotation). Le logiciel WEGO version 2.0 a été utilisé pour regrouper les différents gènes de résistance selon leurs fonctions biologiques dans l'organisme.

Tableau 1: Information sur les amorces des allèles spécifiques des 9 marqueurs SNPs convertis.

\begin{tabular}{|c|c|c|c|c|c|c|}
\hline $\begin{array}{l}\text { Nom du } \\
\text { marqueur }\end{array}$ & $\begin{array}{l}\text { Position } \\
\text { (cM) }\end{array}$ & $\begin{array}{l}\text { Type } \\
\text { d'amorce }\end{array}$ & $\begin{array}{l}\text { Taille du } \\
\text { fragment }\end{array}$ & SNP & $\begin{array}{l}\text { Source } \\
\text { des allèles }\end{array}$ & Séquences des amorces (5' à 3') \\
\hline A2_03535R & 41,15 & $\begin{array}{l}\text { Allèle } \\
\text { flanquant }\end{array}$ & & & & AAGAACATCCAGGGTCAGTG \\
\hline A2_03535F1 & & $\begin{array}{l}\text { Allèle- } \\
\text { spécifique } \\
\text { Allèle- }\end{array}$ & 205 & G & $\mathrm{KN}-1$ & $\begin{array}{l}\text { ATCAAGAAAGAAGGGTCAAGCTCCTG } \\
\text { ATTACTACTAGACGGGAAAGAAGGGT }\end{array}$ \\
\hline A2_03535F2 & & $\begin{array}{l}\text { spécifique } \\
\text { Allèle }\end{array}$ & 215 & $\mathbf{A}$ & Tiligré & CAAGCTCGTA \\
\hline A2_04189R & O & flanquant & & & & TGAAAGTAGCAATGCCTACCC \\
\hline A2_04189F1 & & $\begin{array}{l}\text { Allèle- } \\
\text { spécifique } \\
\text { Allèle- }\end{array}$ & 229 & $\mathbf{G}$ & $\mathrm{KN}-1$ & $\begin{array}{l}\text { ACCATACATTACATAATTGCACATGCT } \\
\text { ATGAAATGAG }\end{array}$ \\
\hline A2_04189F2 & & $\begin{array}{l}\text { spécifique } \\
\text { Allèle }\end{array}$ & 219 & $\mathbf{T}$ & Tiligré & ATCAAATTGCACATGCTATGAAATCAT \\
\hline A2_13136R & 35,90 & flanquant & & & & ATGCAGTCAAGCGGATTTTC \\
\hline A2_13136F1 & & $\begin{array}{l}\text { Allèle- } \\
\text { spécifique }\end{array}$ & 213 & $\mathbf{T}$ & $\mathrm{KN}-1$ & $\begin{array}{l}\text { GAAGAAAGGTGGGAGAGCTGTGGACA } \\
\text { CAGTGAGAGTT }\end{array}$ \\
\hline A2_13136F2 & & $\begin{array}{l}\text { Allèle- } \\
\text { spécifique } \\
\text { Allèle }\end{array}$ & 203 & $\mathbf{C}$ & Tiligré & $\begin{array}{l}\text { CGAGCAGCTGTGGACACAGTGAGACT } \\
\text { C }\end{array}$ \\
\hline A2_21071R & 22,84 & flanquant & & & & CCCAATGTTCAGTTTCTAACCTTT \\
\hline A2_21071F1 & & $\begin{array}{l}\text { Allèle- } \\
\text { spécifique }\end{array}$ & 180 & $\mathbf{G}$ & Tiligré & $\begin{array}{l}\text { ATCAATCGTGTGATAGAAAAGAAGTT } \\
\text { G }\end{array}$ \\
\hline A2_21071F2 & & $\begin{array}{l}\text { Allèle- } \\
\text { spécifique }\end{array}$ & 190 & $\mathbf{A}$ & $\mathrm{KN}-1$ & $\begin{array}{l}\text { ATTACTACTAGACGGTCGTGTGATAGA } \\
\text { AAAGAAGATA }\end{array}$ \\
\hline A2_32056F & 11,97 & $\begin{array}{l}\text { Allèle } \\
\text { flanquant }\end{array}$ & & & & AATGTGGGGTAATGCAAGGA \\
\hline A2_32056R1 & & $\begin{array}{l}\text { Allèle- } \\
\text { spécifique } \\
\text { Allèle- }\end{array}$ & 195 & $\mathbf{G}$ & Tiligré & $\begin{array}{l}\text { GAAGAAAGGTGGGAGAGCAAAAATC } \\
\text { ACATAAGCAGTC } \\
\text { ATCAAAGCAAAAATCACATAAGCACT }\end{array}$ \\
\hline A2_32056R2 & & $\begin{array}{l}\text { spécifique } \\
\text { Allèle }\end{array}$ & 185 & $\mathbf{A}$ & $\mathrm{KN}-1$ & $\mathrm{~T}$ \\
\hline A2_00591F & 27,40 & flanquant & & & & TGTTGGGTTGAGAAGGTGCT \\
\hline A2_00591R1 & & $\begin{array}{l}\text { Allèle- } \\
\text { spécifique } \\
\text { Allèle- }\end{array}$ & 226 & $\mathbf{G}$ & $\mathrm{KN}-1$ & $\begin{array}{l}\text { TATATATAATAGGTCACAACCACAAC } \\
\text { ATCC }\end{array}$ \\
\hline A2_00591R2 & & spécifique & 216 & $\mathbf{T}$ & Tiligré & AGGTCACAACCACAACAACA \\
\hline
\end{tabular}




\begin{tabular}{|c|c|c|c|c|c|c|}
\hline A2_01793R & 21,42 & $\begin{array}{l}\text { Allèle } \\
\text { flanquant }\end{array}$ & & & & СТCACAAACAGCТCСАCСАA \\
\hline A2_01793F1 & & $\begin{array}{l}\text { Allèle- } \\
\text { spécifique }\end{array}$ & 240 & $\mathbf{G}$ & $\mathrm{KN}-1$ & $\begin{array}{l}\text { TATATAAATAATTGTTTGGGTGTGGAA } \\
\text { AGG }\end{array}$ \\
\hline A2_01793F2 & & $\begin{array}{l}\text { Allèle- } \\
\text { spécifique }\end{array}$ & 230 & A & Tiligré & ATTGTTTGGGTGTGGAATGA \\
\hline A2_02471F & $\mathbf{3 4 , 4 0}$ & $\begin{array}{l}\text { Allèle } \\
\text { flanquant } \\
\text { Allèle- }\end{array}$ & & & & TTGGCAGGTTCATTGGATTT \\
\hline A2_02471R1 & & $\begin{array}{l}\text { spécifique } \\
\text { Allèle- }\end{array}$ & 315 & G & Tiligré & $\begin{array}{l}\text { AGTACATGCGTGACAACCGC } \\
\text { ATATAACTTAAGTACATGCGTGACAA }\end{array}$ \\
\hline A2_02471R2 & & $\begin{array}{l}\text { spécifique } \\
\text { Allèle }\end{array}$ & 295 & $\mathbf{A}$ & $\mathrm{KN}-1$ & CGGT \\
\hline $\begin{array}{l}\text { A2_14997BF } \\
\text { A2_14997BR }\end{array}$ & 18,05 & $\begin{array}{l}\text { flanquant } \\
\text { Allèle- }\end{array}$ & & & & GAAACTAGGGCTGAGCATCG \\
\hline 1 & & spécifique & 166 & G & Tiligré & TTTAACTGTTCTCCCACGCC \\
\hline $\begin{array}{l}\text { A2_14997BR } \\
2\end{array}$ & & $\begin{array}{l}\text { Allèle- } \\
\text { spécifique }\end{array}$ & 176 & $\mathbf{A}$ & $\mathrm{KN}-1$ & $\begin{array}{l}\text { ATATAGACTATTTAACTGTTCTCCCAC } \\
\text { СCT }\end{array}$ \\
\hline
\end{tabular}

\section{RESULTATS \\ Analyse phénotypique de la résistance à la maladie des taches brunes au sein de la population des 200 individus $F_{2}$}

Les résultats obtenus indiquent que la variété sensible Tiligré a présenté les valeurs de l'indice de sévérité les plus élevées, notamment au $28^{\text {ème }} \mathrm{JAI}$, soit $77,5 \%$. Chez la variété $\mathrm{KN}$ 1 , cette valeur a été de l'ordre de $2,5 \%$ sur toute la période de l'observation. Chez les quatre individus $F_{1}$ testés, aucun symptôme de la maladie n'a été observé. Au niveau des 200 individus $F_{2}$, on a noté une distribution continue (cas d'hérédité polygénique) de la maladie illustrée par la Figure 1. Les résultats des rapports de ségrégation phénotypique de la population $\mathrm{F}_{2}$ étudiée sont présentés dans le Tableau 2. Ces résultats confirment que la résistance à la maladie des taches brunes est sous le contrôle de QTLs dont l'effet additif favorable serait dû à l'allèle du parent résistant.

\section{Confirmation d'un QTL de résistance}

Les résultats de l'analyse QTL chez 138 individus $\mathrm{F}_{2}$ indiquent la présence d'un QTL significatif avec des valeurs du LOD score max supérieures à 2,1 (LOD score seuil, test de permutation). Ce QTL a été détecté dès le $7^{\text {ème }}$ JAI et représente entre 12,30 ( $7^{\text {c̀me }} \mathrm{JAI}$ ) et $55,49 \%$ (28 ${ }^{\text {ème }}$ JAI) du pourcentage de la variation phénotypique expliqué (PVE). Les effets additifs et de dominance du QTL de résistance, déterminés à partir des scores de la maladie, ont varié respectivement de $+0,22$ ( $\left.7^{\text {ème }} \mathrm{JAI}\right)$ à $+1,01$ ( $\left.28^{\text {ème }} \mathrm{JAI}\right)$, et $-0,16$ ( $7^{\text {ème }}$

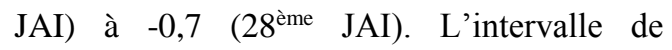
confiance du QTL se situe entre $24,5 \mathrm{cM}$ (7 $7^{\text {ème }}$

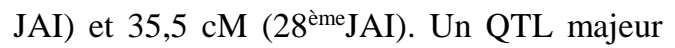
dominant lié à la résistance partielle à la maladie des taches brunes a été détecté notamment aux $21^{\text {ème }}$ et $28^{\text {ème }} \mathrm{JAI}$ pour les scores de la maladie, la sévérité ainsi que l'AUDPC (Tableaux 3 et 4). Les valeurs de PVE et LODs scores de ce QTL sont nettement supérieures et sont de l'ordre de 51,491\% (LOD 22,73) et 55,3314\% (LOD 24,42), respectivement aux $21^{\text {ème }}$ et $28^{\text {ème }}$ JAI (Tableau 4). Parmi les neuf marqueurs testés, les marqueurs A2_21071, A2_00591 et A2_02471 (Figure 2) ont été les plus fortement liés au QTL de résistance, avec des PVE respectivement de l'ordre de 38,36\% (LOD 16,54), 57,66\% (LOD 22,75), 48,81\% (LOD $20,08)$ au $28^{\text {ème }}$ JAI. Les résultats indiquent également un effet additif élevé du QTL de résistance lié à l'allèle favorable du parent $\mathrm{KN}$ 1 notamment aux $21^{\text {ème }}$ et $28^{\text {ème }} \mathrm{JAI}$ ainsi que pour l'AUDPC. La Figure 4 donne les LOD scores maximum, les valeurs des PVE et les effets (additif et de dominance) du QTL détecté 
en fonction du paramètre de résistance utilisé. Les distances cartographiques entre les marqueurs SNPs et le QTL de résistance couvrent une région de $254,1 \mathrm{cM}$ et $246,2 \mathrm{cM}$, respectivement avec la population initiale (200 $F_{2}$ ) et la population finale $\left(138 F_{2}\right)$. Les corrections apportées ont permis d'améliorer, d'une part, les valeurs du PVE et du LOD score et, d'autre part, la précision de la région couverte, soit une réduction de $7,9 \mathrm{cM}$ (Figure 3). La Figure 4 indique les positions et les effets du QTL qBBDR2.1 détecté à partir de chacun des quatre paramètres de résistance chez les 138 individus $F_{2}$. Les résultats montrent également que le QTL détecté avec les scores de la maladie, la sévérité et l'AUDPC est situé à la même position sur la carte génétique soit à $33 \mathrm{cM}$ et entre les marqueurs flanquant A2_00591 et A2_02471.

Région génomique et potentiels gènes de résistance impliqués dans la résistance partielle à la souche Ccap-SA de Colletotrichum capsici.

Deux régions génomiques sur le chromosome 2 renfermant des gènes de résistance susceptibles d'être liés à la maladie des taches brunes du niébé ont été identifiées. La première région, limitée par les marqueurs flanquant A2_21071 et A2_02471, a été identifiée entre le $7^{\text {ème }}$ et le $28^{\text {ème }}$ JAI. Cette région génomique est constituée de 170 complexes de gènes composés de 242 loci. Les complexes de gènes "Leucine-rich repeat protein kinase family protein" et " $L R R$ and $N B$ $A R C$ domains-containing disease resistance protein" sont les plus représentatives avec respectivement 12 et 9 loci.

La seconde région, limitée par les marqueurs flanquant A2_00591 et A2_02471, a été identifiée entre le $21^{\text {ème }}$ et $28^{\text {ème }}$ JAI. L'analyse de cette région a révélé la présence de 100 familles de gènes composées de 133 loci, susceptibles d'être liés à la résistance partielle à $C$. capsici. Parmi ces gènes de résistance, le complexe " $L R R$ and $N B-A R C$ " et le domaine " $N B-A R C$ " sont les plus représentatifs, avec respectivement 8 et 6 loci.

L'annotation fonctionnelle des gènes dans la région génomique limitée par les marqueurs flanquant A2_21071 et A2_02471 à l'aide du programme WEGO a permis de répertorier 29 gènes d'ontologie dont au moins 20 sont associés à la résistance aux maladies ou à la résistance supposée aux maladies. Ces gènes ont été regroupés en trois catégories en fonction de leur action sur les composants cellulaires auxquels ils s'appliquent (CC), des fonctions moléculaires qu'ils réalisent dans l'organisme (MF) et des processus biologiques (BP) dans lesquels ils interviennent (Figure 5).

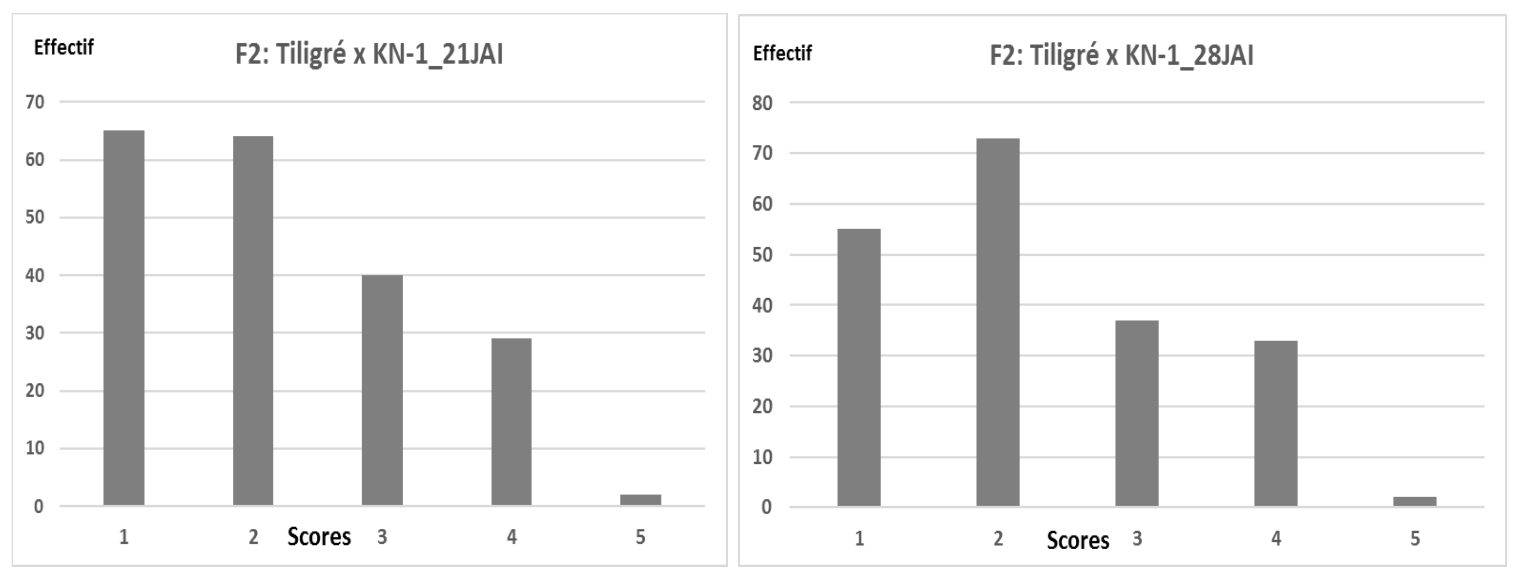

Figure 1: Distribution des scores de la maladie des taches brunes aux $21^{\text {ème }}$ et $28^{\text {ème }} \mathrm{JAI}$ au sein de la population $\mathrm{F}_{2}$. 
Tableau 2: Résultats des rapports de ségrégation selon l'indice de sévérité des individus $F_{2}$ et valeurs de khi-carré (Kh2) pour les données du $28^{\text {ème }} \mathrm{JAI}$.

\begin{tabular}{lllllll}
\hline Population & Npt & $\mathbf{R}$ & $\mathbf{S}$ & Kh2 (1:1) & Kh2 (3:1) & Kh2 (15:1) \\
\hline Tiligré $(\mathbf{S})$ & 10 & 0 & $\mathbf{1 0}$ & - & - & - \\
KN-1 (R) & 10 & $\mathbf{1 0}$ & 0 & - & - & - \\
F $_{\mathbf{1}}$ (S x R) & 4 & $\mathbf{4}$ & 0 & - & - & - \\
F__28JAI $_{\text {28d }}$ & $\mathbf{2 0 0}$ & $\mathbf{1 2 9}$ & $\mathbf{7 1}$ & 16,8 & 11,8 & 292,0 \\
\hline F__28JAI & $\mathbf{1 3 8}$ & $\mathbf{8 5}$ & $\mathbf{5 3}$ & 7,42 & 13,23 & 243,53 \\
\hline
\end{tabular}

$\mathrm{S}=$ Parent Sensible, $\mathrm{R}=$ Parent Résistant, $\mathrm{Npt}=$ nombre de plants testé, Kh2 = Khi carré.

Tableau 3: QTL associé à la résistance de $C$. capsici, marqueurs flanquants, LODs, PVE, additivité et position chez 200 individus $\mathrm{F}_{2}$.

\begin{tabular}{|c|c|c|c|c|c|c|c|c|}
\hline QTL & par. de résistance & Ch. & $\begin{array}{l}\text { Position } \\
\text { (cM) }\end{array}$ & Marqueur F_G/D & $\begin{array}{l}\text { LOD } \\
\text { score }^{\text {a }}\end{array}$ & $\operatorname{PVE}(\%)^{\mathrm{b}}$ & Add. ${ }^{c}$ & Dom. ${ }^{d}$ \\
\hline$q B B D R 2.1$ & Score_7 JAI & 2 & 28 & A2_01793/ A2_21071 & 4,3931 & 10,4089 & 0,2203 & $-0,1215$ \\
\hline$q B B D R 2.1$ & Score_14 JAI & 2 & 30 & A2_21071/ A2_00591 & 6,5462 & 14,5847 & 0,3951 & $-0,0367$ \\
\hline$q B B D R 2.1$ & Score_21 JAI & 2 & 34 & A2_00591/ A2_02471 & 20,8919 & $36,4212 *$ & 0,9986 & $-0,4368$ \\
\hline$q B B D R 2.1$ & Score_28 JAI & 2 & 34 & A2_02471 & 22,3715 & $38,0953 *$ & 1,0031 & $-0,5346$ \\
\hline$q B B D R 2.1$ & Incidence_28JAI & 2 & 33 & A2_00591/ A2_02471 & 5,0122 & 11,8391 & 0,2221 & $-0,0024$ \\
\hline$q B B D R 2.1$ & Sévérité_28 JAI & 2 & 34 & A2_00591/ A2_02471 & 21,7799 & 39,9039* & 0,4502 & $-0,038$ \\
\hline qBBDR2.1 & AUDPC & 2 & 34 & A2_00591/ A2_02471 & 17,2144 & $31,9631 *$ & 13,5448 & $-4,9276$ \\
\hline
\end{tabular}

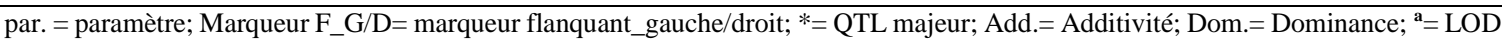
score maximum; ${ }^{b}=$ le pourcentage de variance phénotypique expliqué; ${ }^{c}=$ Les valeurs positives indiquent une augmentation de la sévérité de la maladie (SEV) associée aux allèles de Tiligré et les valeurs négatives indiquent une SEV accrue associée aux allèles de $\mathrm{KN}-1 ;{ }^{\mathrm{d}}=$ Les valeurs positives indiquent que les allèles de Tiligré sont dominants et les valeurs négatives indiquent que les allèles de $\mathrm{KN}-1$ sont dominants.

Tableau 4: QTL associé à la résistance partielle à C. capsici, marqueurs flanquants, LODs, PVE, additivité et position chez 138 individus $\mathrm{F}_{2}$.

\begin{tabular}{|c|c|c|c|c|c|c|c|c|}
\hline$Q T L$ & Fac. de résistance & Ch. & $\begin{array}{l}\text { Position } \\
(\mathrm{cM})\end{array}$ & Marqueur F_G/D & $\begin{array}{l}\text { LOD } \\
\text { score }^{\text {a }}\end{array}$ & $\operatorname{PVE}(\%)^{\mathbf{b}}$ & Add. ${ }^{c}$ & Dom. ${ }^{d}$ \\
\hline$q B B D R 2.1$ & AI & 2 & 27 & 00591 & 3,8632 & ,3009 & 0,2198 & $-0,1642$ \\
\hline$q B B D R 2.1$ & Score_14 JAI & 2 & 28 & A2_21071/ A2_00591 & 6,010 & 20,218 & 0,4175 & $-0,0726$ \\
\hline$q B B D R 2.1$ & Score_21 JAI & 2 & 33 & A2_00591/ A2_02471 & 22,729 & $51,491 *$ & 1,0136 & $-0,6844$ \\
\hline$q B B D R 2.1$ & Score_28 JAI & 2 & 33 & A2_00591/ A2_02471 & 24,4161 & $55,3314 *$ & 1,0132 & $-0,7069$ \\
\hline$q B B D R 2.1$ & Incidence_28JAI & 2 & 31 & A2_00591/ A2_02471 & 4,4217 & 17,7482 & 0,2253 & $-0,0069$ \\
\hline$q B B D R 2.1$ & Sévérité_28 JAI & 2 & 33 & A2_00591/ A2_02471 & 22,0545 & $53,1683^{*}$ & 0,4606 & $-0,0528$ \\
\hline$q B B D R 2.1$ & AUDPC & 2 & 33 & A2_00591/ A2_02471 & 16,9660 & $43,6616 *$ & 13,7328 & $-7,3293$ \\
\hline
\end{tabular}

par. = paramètre; Marqueur F_G/D= marqueur flanquant_gauche/droit; *= QTL majeur; Add.= Additivité; Dom.= Dominance; ${ }^{\mathrm{a}}=$ LOD score maximum; ${ }^{\mathbf{b}}=$ le pourcentage de variance phénotypique expliqué; ${ }^{\mathbf{c}}=$ Les valeurs positives indiquent une augmentation de la sévérité de la maladie (SEV) associée aux allèles de Tiligré et les valeurs négatives indiquent une SEV accrue associée aux allèles de $\mathrm{KN}-1 ;{ }^{\mathrm{d}}=$ Les valeurs positives indiquent que les allèles de Tiligré sont dominants et les valeurs négatives indiquent que les allèles de $\mathrm{KN}-1$ sont dominants. 


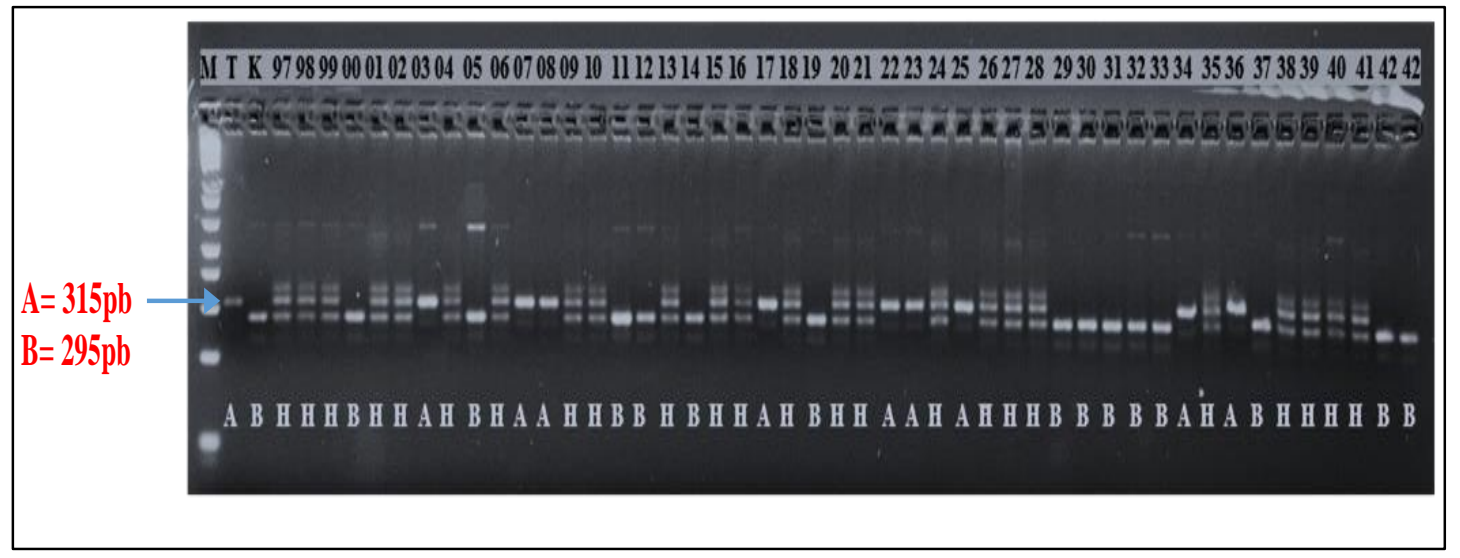

Figure 2: Génotypage à l'aide du marqueur SNP A2_02471 des individus $\mathrm{F}_{2}$.

Tiligré (T), KN-1 (K), A (allèle du parent sensible), B (allèle du parent résistant), H (hétérozygote), gel d'agarose 8\%.

\section{Chr. 2}

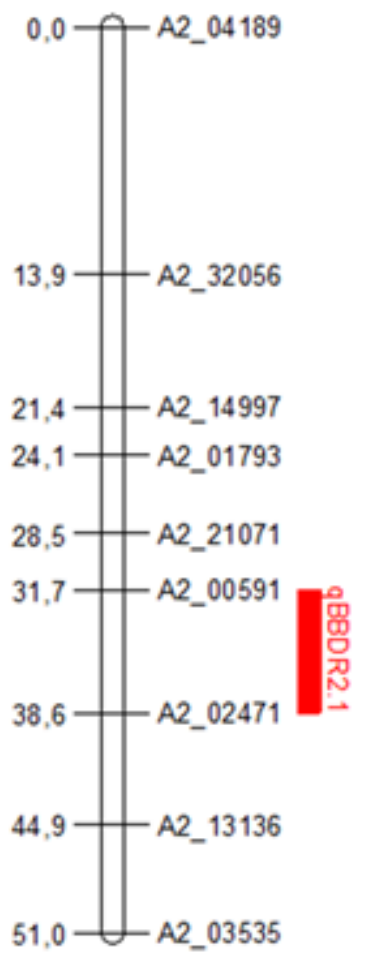

a: $200 \mathrm{~F}_{2}$
Chr. 2

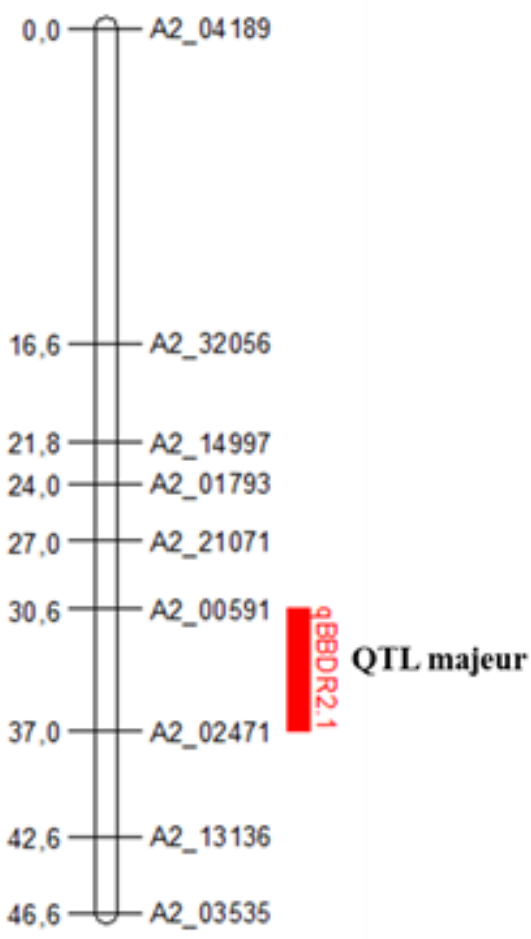

b: $138 \mathrm{~F}_{2}$

Figure 3: Cartographie du QTL de résistance $q B B D R 2.1$ et des marqueurs SNPs entre le $21^{\text {ème }}$ et $28^{\text {ème }}$ JAI. a: cartographie à partir de la population initiale $200 \mathrm{~F}_{2}$; b: cartographie avec 138 individus $\mathrm{F}_{2}$. 


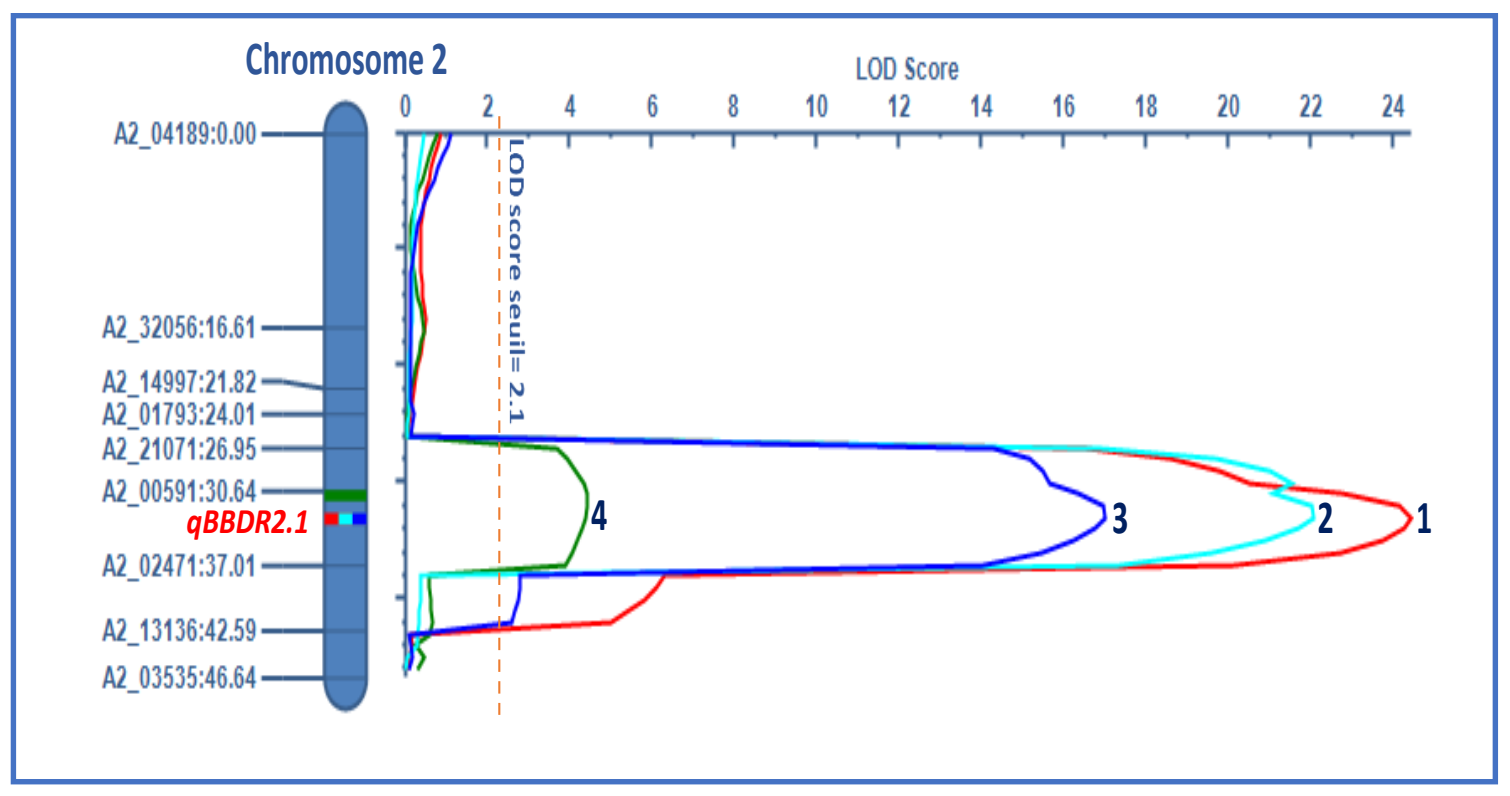

Figure 4: Chromosome 2 et LOD scores max correspondant au QTL $\boldsymbol{q B B D R 2 . 1}$ de résistance détecté entre les marqueurs flanquants A2_21071 et A2_02471 chez 138 individus $\mathrm{F}_{2}$.

$1=$ QTL détecté à partir des scores de la maladie, $2=$ QTL détecté à partir de la sévérité, $3=$ QTL détecté à partir de l'AUDPC, 4 = QTL détecté à partir de l'incidence de la maladie.

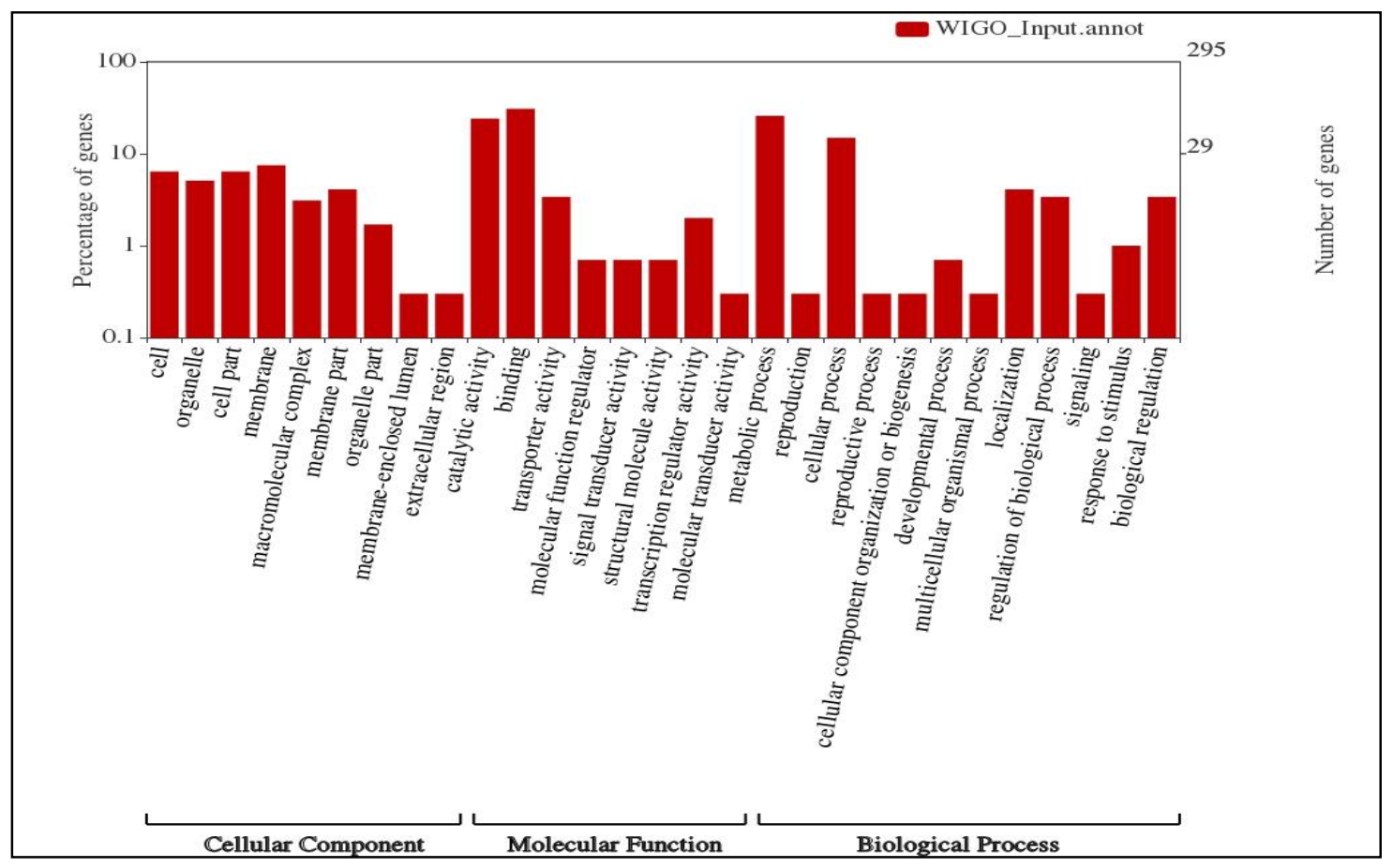

Figure 5: Fonctions biologiques des gènes d'ontologie associés à la maladie des taches brunes du niébé et identifiés dans la région génomique limitée par les marqueurs flanquant A2_21071 et A2_02471. 


\section{DISCUSSION}

La recherche de QTLs et de marqueurs SNPs individuels liés à la résistance du niébé à C. capsici a été d'une très grande utilité. Un QTL majeur dominant, désigné qBBDR2.1 (QTL for brown blotch disease resistance located on chromosome 2 number 1), lié à la résistance partielle à $C$. capsici a été confirmé. Ce QTL a été détecté à partir de 9 marqueurs SNPs polymorphes entre la variété sensible Tiligre et celle résistante $\mathrm{KN}-1$. L'ensemble des 9 marqueurs SNPs a été utilisé pour construire une carte de liaison génétique de la résistance à la maladie des taches brunes. Parmi ces marqueurs, les SNPs A2_21071, A2_00591 et A2_02471 sont les plus fortement liés au QTL de résistance, comparativement aux 7 autres marqueurs. Ces 9 marqueurs qui couvrent une distance de 46,6 cM peuvent être appliqués à des populations biparentales et utilisés en SAM pour la résistance à $C$. capsici. De plus, l'analyse des régions génomiques à partir des marqueurs flanquant nous a permis d'identifier, d'une part, plusieurs gènes potentiellement associés à la résistance partielle à $C$. capsici et, d'autre part, les fonctions possibles de ces gènes de résistance.

Des travaux similaires conduits par Pottorff (2012) avaient permis d'identifier des QTLs et des marqueurs SNPs impliqués dans la résistance du niébé à Macrophomina phaseolina et à Fusarium oxysporum (races 3 et 4). Ces QTLs et marqueurs associés ont été localisés essentiellement sur le chromosome 5. Muchero et al. (2011) ont, quant à eux, identifié des QTLs liés à la résistance du niébé à Macrophomina phaseolina sur le groupe de liaison 7 (chromosome 2, Phytozome). Ohlson et al. (2017) ont aussi identifié et validé un QTL majeur associé au nombre de nodules, à la quantité de nitrogène produite et au poids du plant sec chez le niébé. Ce QTL a été localisé sur le groupe de liaison LG 4 avec un PVE de l'ordre de 49,3\%. Plusieurs QTL de Colletotrichum lindemuthianum ont été signalés sur le chromosome 2 de Phaseolus vulgaris, un parent proche du niébé, ce qui suggère que ce groupe de liaison pourrait jouer un rôle important dans la résistance aux espèces de Colletotrichum (Campa et al., 2014;
Oblessuc et al., 2014; Silva, 2017). Récemment, Ohlson et al. (2018) ont identifié 4 QTLs associés à la résistance aux taches brunes du niébé. Parmi ces QTLs, qBBDR1 est lié aux allèles favorables du parent $\mathrm{KN}-1$ et les trois autres qBBDR2 qBBDR3 et qBBDR4 localisés respectivement sur les chromosomes 3 , 6 et 8 du niébé sont liés aux allèles défavorables du parent sensible Tiligré.

Dans la présente étude, un QTL de résistance à large effet a été confirmé. Les valeurs des PVE et des LODs scores de ce QTL sont très élevés aux $21^{\text {ème }}$ et $28^{\text {ème }}$ JAI. Heath (1994), puis Wang et al. (2014) ont démontré que le stade de développement cytologique influencerait positivement le caractère de résistance et favoriserait la détection de QTL. La réduction de la taille de la population (138 $\mathrm{F}_{2}$ ) après l'élimination des génotypes improbables a permis d'améliorer les valeurs du PVE et les effets du QTL (additif et de dominance). Cette correction permet une meilleure appréciation de la distance génétique entre marqueurs adjacents, et donc une carte génétique de bonne qualité (Ferreira et al., 2006). Les effets additifs élevés observés au $21^{\text {ème }}$ JAI $(1,0136)$ et au $28^{\text {ème }}$ JAI $(1,0132)$ indiquent une contribution significative de l'allèle parental mâle (KN-1) à la résistance.

De plus, la détection de QTL à partir de la sévérité de la maladie et de l'AUDPC permet de mieux appréhender l'hérédité de la résistance à la maladie des taches brunes du niébé par rapport à l'incidence de la maladie. En effet, les résultats obtenus avec ces deux paramètres de la résistance sont très proches de ceux obtenus avec les scores de la maladie. Les valeurs du PVE et du LODs score ainsi que la position du QTL, sont nettement améliorées et précises.

Il ressort donc de nos travaux que le choix du mode de désignation des individus en classes de sensibilité est très déterminant dans la détection des QTLs et dans l'étude de l'hérédité des caractères monogéniques. La classification selon l'indice de sévérité pourra être adoptée pour l'étude de l'hérédité de la résistance.

En considérant le QTL de résistance détecté, deux régions génomiques localisées 
sur le chromosome 2 ont été identifiées et sont impliquées dans la résistance à la maladie des taches brunes du niébé. Ces régions génomiques renferment plusieurs complexes de gènes riches en des domaines comme " $N B$ $A R C$ ", "LRR et $N B-A R C$ " et " $L R R$ ". Les travaux de Ooijen et al. (2008) ont montré le rôle très important que jouent les domaines "NB-ARC" et "LRR et NB-ARC" dans la résistance aux maladies chez plusieurs espèces végétales. Deux gènes de résistance $\mathrm{R}$ contenant le domaine "NB-ARC" ont été associés à l'anthracnose chez le sorgho (Upadhyaya et al., 2013). Chez le tournesol, Darvishzadeh (2007) a identifié des régions génomiques renfermant les gènes codant pour la "phenylalanine ammonia-lyase 2 (PAL2)" et la thaumatine, qui seraient impliqués dans la résistance à Phoma macdonaldi.

\section{Conclusion}

L'utilisation de QTL et de marqueurs associés dans les programmes d'amélioration variétale du niébé constitue un véritable défi pour les sélectionneurs de cette culture. Un QTL majeur dominant et des marqueurs SNPs liés à la résistance partielle du niébé à l'isolat C.cap-SA de C. capsici ont respectivement été confirmé et validés. La confirmation de QTL est une étape très importante dans le processus de sa validation. C'est une étape qui permet d'améliorer la position (intervalle de confiance) du QTL, d'optimiser ses effets et de tester sa stabilité. Le QTL de résistance confirmé est lié à l'allèle favorable du parent $\mathrm{KN}-1$ et pourra être introgressé à d'autres variétés de niébé pour être validé. L'identification de marqueurs flanquant la région génomique et liés au QTL de résistance devrait contribuer à un repérage des gènes de résistance, et donc une meilleure compréhension des mécanismes de défense de la plante. Cela permettra d'exploiter le clonage de ces gènes de résistance dans des programmes de pyramidages de gènes dans le cas d'un complexe de pathogènes.

\section{CONFLIT D'INTERETS}

Les auteurs déclarent ne pas avoir de conflit d'intérêts.

\section{CONTRIBUTIONS DES AUTEURS}

MT et EWO nous ont reçus au laboratoire Timko Lab aux USA pour notre stage de perfectionnement. Ils ont en outre contribué à la conception des amorces SNPs et à l'analyse des données bio-informatiques. EPZ nous a permis d'obtenir les souches de Colletotrichum capsici utilisées pour les différentes inoculations, a contribué à la rédaction du manuscrit. SMZ a supervisé les différents croisements qui ont permis de développer les populations en ségrégation. NO a contribué à la correction du manuscrit. JTTB est le PI du projet sous lequel les activités de recherches ont été menées. JO et JBT ont facilité l'obtention d'une bourse pour mon stage aux USA. PS et MS ont codirigé les travaux de Thèse qui ont permis à la rédaction de ce manuscrit. Ils ont aussi contribué à la rédaction du manuscrit.

\section{REMERCIEMENTS}

Ce travail a été rendu possible grâce à l'appui financier de la fondation Kirkhouse Trust et à l'assistance technique de Timko Lab du département de Biologie de l'Université de Virginie (USA). Nous exprimons toute notre reconnaissance au Professeur Mike TIMKO et au Dr Erik Ohlson pour leur nombreux soutien.

\section{REFERENCES}

Adegbite AA, Amusa NA. 2008. The major economic field diseases of cowpea in the humid agro-ecologies of south-western Nigeria. African Journal of Biotechnology, 7(25): 4706-4712. DOI: 10.1080/03235400802677768

Adetumbi JA, Akinyosoye ST, Olowolafe MO, Oloyede-Kamiyo QO, Agbeleye OA. 2016. Genetic linkage map of cowpea (Vigna unguiculata (L) Walp) using SNP markers. African Journal of Biotechnology, 15(20): 830-834. DOI: 10.5897/AJB2015.15167

Begum MM, Sariah M, Puteh AB, Zainal Abidin MA. 2008. Pathogenicity of Colletotrichum truncatum and its Influence on soybean seed quality. International Journal of Agriculture and 
Biology, 10(4): 393-398. DOI: 07443/MFA/2008/10-4-393-398

Bratteler M, Baltisberger M, Widmer A. 2006. QTL analysis of intraspecific differences between two Silene vulgaris ecotypes. Annals of Botany, 98: 411-419. DOI:10.1093/aob/mcl113

Campa A, Rodríguez-Suárez C, Giraldez R, Ferreira JJ. 2014. Genetic analysis of the response to eleven Colletotrichum lindemuthianum races in a RIL population of common bean (Phaseolus vulgaris L.). BMC Plant Biology, 14(1): 114-115.

DOI:

https://doi.org/10.1186/1471-2229-14115

Churchill GA, Doerge RW. 1994. Empirical threshold values for quantitative trait mapping. Genetics, 138: 963-971. DOI: 10.1093/genetics/138.3.963

Collard BCY, Jahufer MZZ, Brouwer JB, Pang ECK. 2005. An introduction to markers, quantitative trait loci (QTL) mapping and marker-assisted selection for crop improvement: the basic concepts. Euphytica, 142(1-2): 169-196. DOI: 10.1007/s10681-005-1681-5

Collard BCY, Jolley R, Bovill WD, Grams RA, Wildermuth GB, Sutherland MW. 2006. Confirmation of QTL mapping and marker validation for partial seedling resistance to crown rot in wheat line 2-49. Australian Journal of Agricultural Research, 57(9): 967-973. DOI: http://dx.doi.org/10.1071/AR05419

Dabat M-H, Lahmar R, Guissou R. 2012. La culture du niébé au Burkina Faso: Une voie d'adaptation de la petite agriculture à son environnement. Autrepart, 62(3): 95. DOI: 10.3917/autr.062.0095

Darvishzadeh R. 2007. Déterminisme génétique de la résistance du tournesol au Phoma. Thèse de Doctorat de l'Institut National Polytechnique de Toulouse, Institut National Polytechnique de Toulouse, Toulouse, $201 \mathrm{p}$.

Doyle JJ, Doyle JL. 1987. A rapid DNA isolation procedure for small quantities of fresh leaf tissue. Phytochemical Bulletin, 19(1): 11-15.
Enyiukwu DN, Awurum AN, Ononuju CC, Nwaneri JA. 2014. Biology and management strategies of cowpea anthracnose disease caused by Colletrotrichum species. Greener Journals of Biochemistry and Biotechnology, 1(2): 052-065. DOI: 10.15580/GJBB.2014.2.070414288

FAOSTAT. 2017. Production Végétale, Quantité Produite. FAOSTAT, Rome, Italie. https://faostat.fao.org.

Ferreira A, da Silva MF, da Costa e Silva L, Cruz CD. 2006. Estimating the effects of population size and type on the accuracy of genetic maps. Genetics and Molecular Biology, 29(1): 187-192. DOI: https://doi.org/10.1590/S141547572006000100033

Gaudet M, Fara A-G, Beritognolo I, Sabatti M. 2009. Allele-Specific PCR in SNP Genotyping. In Single Nucleotide Polymorphisms, Methods in Molecular Biology 578, Komar AA (ed). Springer Science+Business Media: USA; 415424. DOI: $10.1007 / 978-1-60327-411-$ 1_26.

Halime MH, Belko N, Cisse N, Sine B, Ndoye I. 2014. Amélioration de l'adaptation à la sécheresse chez le niébé (Vigna unguiculata L. Walpers). Journal of Applied Biosciences, 77: 6550-6563. DOI: $10.4314 /$ jab.v77i1.12

Heath MC. 1994. Genetics and cytology of age-related resistance in North American cultivars of cowpea (Vigna unguiculata) to the cowpea rust fungus (Uromyces vignae). Canadian Journal of Botany, 72: 575-581. DOI: https://doi.org/10.1139/b94-076

INERA. 2003. Plan stratégique de la recherche sur les oléagineux annuels et les légumineuses à graines (OléoProtéagineux). INERA, Programme Oléagineux, $56 \mathrm{p}$.

Muchero W, Ehlers JD, Close TJ, Roberts PA. 2011. Genic SNP markers and legume synteny reveal candidate genes underlying QTL for Macrophomina phaseolina resistance and Maturity in Cowpea [Vigna unguiculata (L) Walp.]. 
BMC Genomics BioMed., 12(8): 14. DOI: https://doi.org/10.1186/1471-2164-12-8

Nedumaran S, Abinaya $\mathrm{P}$, Jyosthnaa $\mathrm{P}$, Shraavya B, Rao P, Bantilan C. 2015. Grain legumes production, consumption and trade trends in developing countries. ICRISAT Research Program, 502(60): 47.

Obi VI, Barriuso-Vargas JJ. 2014. Situation of biofungicides reconnaissance, a case of anthracnose disease of cowpea. American Journal of Plant Sciences, 5: 1202-1211. DOI:

http://dx.doi.org/10.4236/ajps.2014.5913 3

Oblessuc PR, Baroni RM, Pereira GDS, Chiorato AF, Carbonell SAM, Brinez B, Silva LDCE, Garcia AAF, Camargo LEA, Kelly JD, Benchimol-Reis LL. 2014. Quantitative analysis of racespecific resistance to Colletotrichum lindemuthianum in common bean. Molecular Biology, 34: 1313-1329. DOI 10.1007/s11032-014-0118-z

Ohlson EW, Seido SL, Suheb M, Santos CAF, Timko MP. 2017. QTL mapping of ineffective nodulation and nitrogen utilization-related traits in the IC-1 mutant of cowpea. Crop Science, 58: 9. DOI: $10.2135 /$ cropsci2017.07.0439

Ohlson EW, Thio IG, Sawadogo M, Sérémé P, Timko MP. 2018. Quantitative trait loci analysis of brown blotch resistance in cowpea variety KN1. Molecular Breeding, 38(110): 1-8. DOI: https://doi.org/10.1007/s11032-0180867-1

Ooijen GV, Mayr G, Kasiem MMA, Albrecht M, Cornelissen BJC, Takken FLW. 2008. Structure-function analysis of the NBARC Domain of plant disease resistance proteins. Journal of Experimental Botany, 59(6): $1383-1397 . \quad$ DOI: $10.1093 / \mathrm{jxb} / \mathrm{ern} 045$

Ouédraogo S. 2003. Impact économique des variétés améliorées du niébé sur les revenus des exploitations agricoles du plateau central du Burkina Faso. Tropicultura, 21(4): 204-210.
Pottorff MO. 2014. Genetic mapping of Fusarium oxysporum F.sp. Tracheiphilum Race 3 and Race 4, Macrophomina phaseolina resistance and other traits in cowpea (Vigna unguiculata [L.] Walp). PhD Thesis, University of California Riverside, California, $304 \mathrm{p}$.

Sérémé P, Diasso GA, Ake S. 2001. Mise en évidence au Burkina Faso d'un nouveau taxon de Colletotrichum dans l'étiologie de la maladie des taches brunes du niébé. Tropicultura, 19(3): 156-160.

Shaner G, Finney RE. 1977. The effect of nitrogen fertilization on the expression of slow-mildewing resistance in Knox wheat. Phytopathology, 67: 1051-1056. DOI: http://dx.doi.org/10.1094/Phyto-671051

Silva LCD. 2017. Linkage fine-mapping, Gwas and QTLs affecting morpho-agronomic traits of a common bean RIL. PhD Thesis, Universidade Federal de Viçosa, Viçosa, $118 \mathrm{p}$.

Thio IG. 2018. Etude génétique de la résistance du niébé (Vigna unguiculata (L.) Walp.) à la maladie des taches brunes causée par Colletotrichum capsici (Syd.) Butler et Bisby au Burkina Faso. Thèse Unique de Doctorat, Université Ouaga Pr Joseph KIZERBO, Burkina Faso, 211 p.

Upadhyaya HD, Wang Y-H, Sharma R, Sharma S. 2013. Identification of genetic markers linked to anthracnose resistance in Sorghum using association analysis. Theor. Appl. Genet., 126: 1649-1657. DOI: $\quad$ https://doi.org/10.1007/s00122013-2081-1

Voorrips RE. 2002. MapChart: Software for the graphical presentation of linkage maps and QTLs. Journal of Heredity, 93(1): $\quad 77-78 . \quad$ DOI: https://doi.org/10.1093/jhered/93.1.77

Wang X, Mace E, Hunt C, Cruickshank A, Henzell R, Parkes H. 2014. Two distinct classes of QTL determine rust resistance in Sorghum. BMC Plant Biology, 14(1): $1-14$.

DOI: https://doi.org/10.1186/s12870-0140366-4 